PAPER • OPEN ACCESS

Boosting the performance of Brillouin amplification at sub-quarter-critical densities via reduction of parasitic Raman scattering

To cite this article: R M G M Trines et al 2021 Plasma Phys. Control. Fusion 63124003

View the article online for updates and enhancements.
You may also like

- $\frac{\text { Parametrization of current-voltage }}{\text { characteristics and operation domains of }}$
$\frac{\text { cylindrical emissive probes in collisionless }}{\text { Maxwellian plasmas at rest }}$
$\frac{\text { S Shahsavani, X Chen and G Sanchez- }}{\text { Arriaga }}$
- Simultaneous specific heat and thermal
$\frac{\text { conductivity measurement of individual }}{\text { nanostructures }}$
Jianlin Zheng, Matthew C Wingert, Jaeyun
Moon et al.
- Robust real-time feedback algorithms for
plasma kinetic control in advanced
$\frac{\text { tokamak scenarios }}{\text { S Wang, D Moreau, E Witrant et al. }}$




\title{
Boosting the performance of Brillouin amplification at sub-quarter-critical densities via reduction of parasitic Raman scattering
}

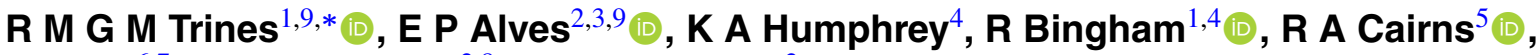

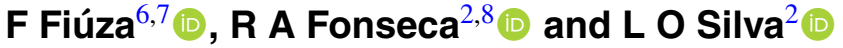 \\ ${ }^{1}$ Central Laser Facility, STFC Rutherford Appleton Laboratory, Didcot, OX11 0QX, United Kingdom \\ ${ }^{2}$ GoLP/Instituto de Plasmas e Fusão Nuclear, Instituto Superior Técnico, Universidade de Lisboa, \\ 1049-001 Lisbon, Portugal \\ ${ }^{3}$ Department of Physics and Astronomy, University of California Los Angeles, Los Angeles, CA 90095, \\ United States of America \\ ${ }^{4}$ SUPA, Department of Physics, University of Strathclyde, Glasgow G4 0NG, United Kingdom \\ ${ }^{5}$ University of St Andrews, St Andrews, Fife, KY16 9SS, United Kingdom \\ ${ }^{6}$ Lawrence Livermore National Laboratory, Livermore, CA, United States of America \\ ${ }^{7}$ SLAC National Accelerator Laboratory, Menlo Park, CA 94025, United States of America \\ ${ }^{8}$ DCTI/ISCTE Lisbon University Institute, 1649-026 Lisbon, Portugal \\ E-mail: raoul.trines@stfc.ac.uk
}

Received 12 April 2021, revised 7 September 2021

Accepted for publication 5 October 2021

Published 25 October 2021

\begin{abstract}
Brillouin amplification of laser pulses in plasma has been shown to be a promising approach to produce picosecond pulses of petawatt power. A key challenge is preservation of the quality of the amplified pulse, which requires control of parasitic instabilities that accompany the amplification process. At high plasma densities $\left(>_{c r} / 4\right)$, ponderomotive filamentation has been identified as the biggest threat to the integrity of the amplifying pulse. It has therefore been proposed to perform Brillouin scattering at densities below $n_{c r} / 4$ to reduce the influence of filamentation. However, parasitic Raman scattering can become a problem at such densities, contrary to densities above $n_{c r} / 4$ where it is forbidden. In this paper, we investigate the influence of parasitic Raman scattering on Brillouin amplification at densities below $n_{c r} / 4$. We expose the specific problems posed by both Raman backward and forward scattering, and how both types of scattering can be mitigated, leading to an increased performance of the Brillouin amplification process.
\end{abstract}

Keywords: Brillouin amplification, laser-plasma interaction, parametric instabilities, high energy density physics

(Some figures may appear in colour only in the online journal)

9 Authors R Trines and E Alves contributed equally to this work.
* Author to whom any correspondence should be addressed. 


\section{Introduction}

Plasma-based laser amplification offers a promising route to producing next-generation laser powers and intensities [1-7]. The amplification process exploits the parametric instabilities of Raman and Brillouin scattering in plasma. In these two processes, two electromagnetic waves at slightly different frequencies propagating in plasma in opposite directions exchange energy via a plasma wave. For Raman scattering, this is a fast electron plasma wave, while for Brillouin scattering it is a slower ion-acoustic wave [8]. When it comes to laser beam amplification, Raman and Brillouin scattering have different properties and serve different purposes $[6,7]$. Raman amplification yields the shortest output pulses and the highest amplification ratios, but it is sensitive to fluctuations in the experimental parameters and requires high accuracy in the matching of laser and plasma frequencies. Brillouin amplification yields lower peak intensities or amplification ratios, but is far more robust to parameter fluctuations or frequency mismatch, more efficient (as less laser energy stays behind in the plasma wave) and more suitable for the production of pulses with a high total power or energy. Also, in the so-called 'strong-coupling' regime of Brillouin amplification, where the laser intensities are so high that the ion plasma wave is a driven mode rather than a resonant mode $[7,8]$, the duration of the compressed pulse can be shortened until it becomes comparable to that for Raman amplification [7, 9].

In recent years, substantial progress has been made in both experiments [10-16] and modelling [17-26] of plasma-based Raman and Brillouin amplification. In order to turn these processes into practical schemes for the production of high-power laser pulses, controlling the quality of the amplified pulse (smooth envelope, low-intensity prepulse) is as important as maximising its power and energy. This involves reduction and control of competing instabilities, such as pump Raman backscattering, seed Raman forward scattering (RFS), filamentation and modulational instabilities. Previous investigations into Raman and Brillouin scattering identified filamentation as the most important limiting factor for successful amplification $[9,17]$. This is especially true for Brillouin amplification, since it employs higher plasma densities than Raman amplification. Originally, it was proposed to conduct Brillouin amplification experiments at $n_{0} / n_{c r}=0.3$ [7], where $n_{0}$ denotes the background plasma electron density and $n_{c r}$ denotes the critical density for the wave length of the pump laser. At these high densities, all Raman scattering is forbidden [8], but instabilities like ponderomotive filamentation are strongly boosted [9]. In more recent experimental and numerical work [14-16, $22,24,27,28]$, the plasma density for Brillouin amplification has been reduced from $n_{0} / n_{c r}=0.3$ to $0.05 \leq n_{0} / n_{c r} \leq$ 0.15 . While this reduces filamentation, Raman instabilities will return with a vengeance for any $n_{0} / n_{c r}<0.25$ [8], and can be expected to interfere with the Brillouin amplification process [29]. Examples of strong longitudinal pulse envelope modulations and intense prepulses preceding the amplified probe pulse, all induced by RFS, have been observed before [28]. Various strategies have been proposed to reduce the influence of Raman scattering, such as chirping the pump laser beam [23, 25] or adding a longitudinal magnetic field [30, 31].

In this paper, we aim to investigate the influence of stimulated Raman scattering (both backward and forward) on Brillouin amplification at sub-quarter-critical densities $\left(n_{0} / n_{c r}<\right.$ $0.25)$. This will be done as follows. First, we will give a summary of the self-similar theory of Brillouin amplification in the strong-coupling regime [7, 9]. Next, we will thoroughly analyse the results of a particle-in-cell (PIC) simulation of a scenario where strong SRS is likely to occur: laser beam intensities of $10^{16} \mathrm{~W} \mathrm{~cm}^{-2}$ for a wavelength of $1 \mu \mathrm{m}$ and $n_{0} / n_{c r}=0.05$. Finally, we will carry out an extensive parameter scan to identify the parameters for the pump laser and the plasma column where the best results (highest amplification factor, lowest relative level of parasitic SRS) can be obtained. We will also investigate and discuss the impact of using non-constant plasma density profiles, as proposed by Riconda et al [28].

\section{Self-similar theory of Brillouin amplification}

To explore how the intensity and duration of a Brillouinamplified probe pulse can be controlled, we use the selfsimilar model of Andreev et al [7] for Brillouin amplification in the strong-coupling regime (high pump intensity). Further details regarding this model can also be found in Lehmann and Spatschek [22] or Trines et al [26]. We start from a homogeneous plasma with electron number density $n_{0}$, plasma frequency $\omega_{p}^{2}=e^{2} n_{0} /\left(\varepsilon_{0} m_{e}\right)$, ion plasma frequency $\omega_{p i}=\omega_{p} \sqrt{Z m_{e} / m_{i}}$, electron/ion temperatures $T_{e}$ and $T_{i}$, electron thermal speed $v_{T}^{2}=k_{B} T_{e} / m_{e}$, Debye length $\lambda_{D}=$ $v_{T} / \omega_{p}$, and a pump laser pulse with wave length $\lambda$, intensity $I$, frequency $\omega_{0}=2 \pi c / \lambda$, dimensionless amplitude $a_{0} \equiv$ $8.55 \times 10^{-10} \sqrt{g} \sqrt{I \lambda^{2}\left[\mathrm{Wcm}^{-2} \mu \mathrm{m}^{2}\right]}$, where $g=1 \quad(g=1 / 2)$ denotes linear (circular) polarisation, and wave group speed $v_{g} / c=\sqrt{1-\omega_{p}^{2} / \omega_{0}^{2}}=\sqrt{1-n_{0} / n_{c r}}$. To ensure we are in the strong-coupling regime, we need to choose a large pump amplitude: $a_{0}^{2}>8 g\left(\omega_{0} / \omega_{p e}\right)^{2}\left(v_{T} / c\right)^{3} \sqrt{Z m_{e} / m_{i}}$ [8]. From Trines et al [26], we also learn that $a_{0}$ must be sufficiently large so the sc-Brillouin linear growth rate is larger than any damping coefficient (collisional damping, Landau damping on ions) in the system. However, for a low-density plasma with low collisionality, this will be ensured by default by the large amplitudes that we need for strongly coupled Brillouin scattering.

Let the durations of pump and probe pulse be given by $\tau_{0}$ and $\tau_{1}$, and define $\Gamma_{B B S-s c}$ via $\Gamma_{B B S-s c}^{3}=\left(v_{g} / c\right)^{2} \quad \omega_{p i}^{2} \quad \omega_{0} /(2 g)=\omega_{0}^{3}\left(Z m_{e} / m_{i}\right)\left(n_{0} / n_{c r}\right)(1-$ $\left.n_{0} / n_{c r}\right) /(2 g)$, the coupling constant for Brillouin scattering in the strong-coupling regime $[8,26]$. Following our earlier results [26], we obtain the following relations between pulse amplitudes (where $\left\|a_{1}\right\|$ denotes the maximum of the spatiotemporal envelope function $a_{1}(x, t)$ of the seed function) and durations:

$$
\Gamma_{B B S-s c}^{3} a_{0}^{2} \tau_{0} \tau_{1}^{2} \approx 22
$$




$$
\Gamma_{B B S-s c}\left\|a_{1}\right\|^{2 / 3} \tau_{1} \approx(13.8)^{1 / 3} \approx 2.40
$$

This means that the initial probe pulse duration is not a free parameter: equation (2) dictates the optimal initial probe pulse duration $\tau_{\text {opt }}$ for a given initial probe pulse amplitude $a_{1}$. From previous numerical work on Raman [19, 32] and Brillouin amplification [21, 22], it follows that if the probe pulse is too short for its amplitude initially, it will first generate a much longer secondary probe pulse behind the original probe (which does fulfill equation (2)) and this secondary probe will then amplify while the original short probe will hardly gain in intensity. Thus, trying to produce ultra-short laser pulses via Brillouin amplification by reducing the initial pulse duration simply does not work. Earlier attempts in this direction $[27,28]$ showed no increase in total pulse power (as opposed to pulse peak intensity), confirming the results of $[21,22]$. An in-depth discussion of these matters can be found in [26].

We utilize these relationships to guide the design of the initial probe pulse in the numerical experiments presented below. We consider probe pulses with initially the same intensity as the pump, and tune their initial duration as prescribed by equation (2). In this way, we bypass the inefficient linear phase of the amplification process and promptly enter the nonlinear self-similar regime, where the pump beam will be largely depleted and its energy efficiently transferred to the probe.

\section{Parasitic instabilities at sub-quarter-critical densities}

\subsection{Theory}

In order to assess the relative importance of various competing processes, we have calculated the growth rates for Raman backward scattering (RBS), $\gamma_{R B S}=\left(a_{0} / 2\right) \sqrt{\omega_{0} \omega_{p e}}$ [8], RFS [8], $\gamma_{R F S}=\left(a_{0} / 2 \sqrt{2}\right) \omega_{p e}^{2} / \omega_{0}$, ponderomotive filamentation, $\gamma_{P F}=(5 / 4) a_{0} \omega_{p i}$ in the limit $\gamma_{P F} \gg k_{P F} v_{T} \sqrt{m_{e} / m_{i}}$, [36, 37], relativistic filamentation, $\gamma_{R F}=\left(a_{0}^{2} / 8\right) \omega_{p i}^{2} / \omega_{0}$, [38], and strong-coupling Brillouin backward scattering, $\gamma_{B B S-s c}=$ $\Gamma_{B B S-s c} a_{0}^{2 / 3}$, see above, for $10^{-3} \leq n_{0} / n_{c r} \leq 2.5 \times 10^{-1}$ [8]. The pump field amplitude was chosen to be two times the threshold value for strong-coupling Brillouin scattering [8], i.e. $a_{0}=2 a_{0-s c}$ where $a_{0-s c}=2\left(v_{T} / c\right)^{3 / 2}\left(n_{c r} / n_{0}\right)^{1 / 2}(1-$ $\left.n_{0} / n_{c r}\right)^{1 / 4}\left(Z m_{e} / m_{i}\right)^{1 / 4}$. We made this particular choice because we found earlier that the pump pulse amplitude should be as low as possible, but still above the strong-coupling threshold, for optimal results [9]. With these adjustments to the pump intensity, the density dependence of the various growth rates is as follows:

$$
\begin{gathered}
\frac{\gamma_{B B S-s c}}{\omega_{0}}=\sqrt{3} \frac{v_{T}}{c}\left(\frac{m_{e}}{m_{i}}\right)^{1 / 2}\left(2 Z^{2}\right)^{1 / 3}=\text { const. } \\
\frac{\gamma_{R B S}}{\omega_{0}}=2 \sqrt{2}\left(\frac{m_{e}}{m_{i}}\right)^{1 / 4}\left(\frac{\nu_{T}}{c}\right)^{3 / 2}\left(\frac{\omega_{0}}{\omega_{p e}}\right)^{1 / 2} \propto\left(\frac{n_{0}}{n_{c r}}\right)^{-1 / 4},
\end{gathered}
$$

$$
\begin{gathered}
\frac{\gamma_{R F S}}{\omega_{0}}=2\left(\frac{m_{e}}{m_{i}}\right)^{1 / 4}\left(\frac{v_{T}}{c}\right)^{3 / 2} \frac{\omega_{p e}}{\omega_{0}} \propto\left(\frac{n_{0}}{n_{c r}}\right)^{1 / 2}, \\
\frac{\gamma_{P F}}{\omega_{0}}=5\left(\frac{m_{e}}{m_{i}}\right)^{3 / 4}\left(\frac{v_{T}}{c}\right)^{3 / 2}=\text { const. } \\
\frac{\gamma_{R F}}{\omega_{0}}=2\left(\frac{m_{e}}{m_{i}}\right)^{3 / 2}\left(\frac{v_{T}}{c}\right)^{3}=\text { const. }
\end{gathered}
$$

These growth rates are plotted in figure 1; all growth rates are shown relative to the Brillouin scattering growth rate. For our particular configuration, we find that the growth rate for the ponderomotive filamentation does not change with density, while the RBS growth rate increases and the RFS growth rate decreases with decreasing plasma density. As will be confirmed in our simulation results below, a density of $n_{0} / n_{c r}=$ 0.05 is too high, driving too much RFS, while much better results can be obtained for densities around $n_{0} / n_{c r}=0.01$. At even lower densities, e.g. $n_{0} / n_{c r}=0.001$, one has to worry that the growth rate for Brillouin scattering becomes too low for this process to be useful, while the plasma frequency becomes low enough that the (anti-)Stokes side bands of Raman scattering, located at $\omega_{0} \pm \omega_{p e}$, may fall within the bandwidth of the probe pulse and may be directly driven by it. This fixes the useful density interval to roughly $0.005 \leq n_{0} / n_{c r} \leq 0.02$.

It should be noted that the adjusted growth rate of Raman backscatter increases for decreasing plasma density because the pump laser intensity is increased in order to remain above the threshold for strong-coupling Brillouin scattering. However, we do not observe a corresponding increase in the overall level of RBS in our numerical simulations. It is conjectured that RBS saturates at lower densities due to wave breaking of the RBS Langmuir wave, since the amplitude threshold for wave breaking scales as $\left(n_{0} / n_{c r}\right)^{3 / 4}$, while the adjusted pump amplitude scales as $a_{0-s c} \propto\left(n_{c r} / n_{0}\right)^{1 / 2}$.

\subsection{Numerical simulations}

In order to investigate the influence of RBS and RFS on Brillouin amplification, we have carried out 1-D static-window simulations using the PIC code OSIRIS [33-35]. PIC codes like OSIRIS solve the set of Maxwell's curl equations coupled to the relativistic equation of motion for charged particles, thus capturing the self-consistent interplay between the motion of charged plasma particles and their collective electric and magnetic fields. Hence, in the context of this work, OSIRIS simulations provide a first-principles kinetic description of Brillouin amplification, including parasitic instabilities like Raman scattering. We begin by analysing a scenario where the deleterious effects of Raman scattering are expected to be high, at a relatively high plasma density of $n_{0} / n_{c r}=0.05$, and high pulse intensities of $10^{16} \mathrm{~W} \mathrm{~cm}^{-2}$, corresponding to $a_{0} \simeq 0.085 \simeq$ $10 a_{0-s c}$. The plasma column length $L_{p}$ is chosen to be $L_{p}=$ $2900 \mathrm{c} / \omega_{0}=0.46 \mathrm{~mm}$, which corresponds to $\sim 15$ strongcoupling Brillouin growth lengths $\left(v_{g} / \gamma_{B B S-s c}\right)$. The initial probe pulse duration is set to $\tau_{\text {opt }}$, according to equation (2), and the pump duration is $\tau_{\text {pump }}=2 L_{p} / v_{g} \simeq 3.1 \mathrm{ps}$. The results are displayed in figures 2 and 3. 

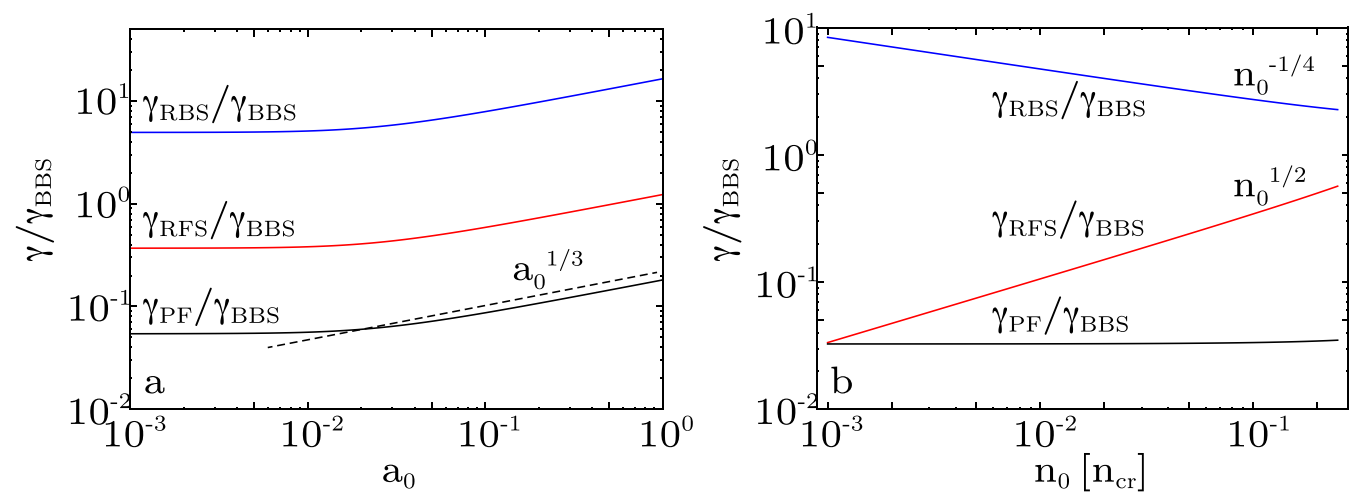

Figure 1. (a) Growth rate of parasitic Raman forward $\left(\gamma_{R F S}\right)$ and back-scattering $\left(\gamma_{R B S}\right)$ and ponderomotive filamentation $\left(\gamma_{P F}\right)$ relative to the Brillouin back-scattering growth rate $\left(\gamma_{B B S}\right)$ verse pump intensity for a plasma density of $n_{0} / n_{c r}=0.05$. We note that here $\gamma_{B B S}$ is given by the numerical solution of the full Brillouin scattering dispersion relation [8], which captures the transition from weak- to strong-coupling regimes (corresponding to the inflection in the plotted curves); in the rest of the text, we use $\gamma_{B B S-s c}$ to refer to the Brillouin growth rate in the asymptotic strong coupling regime. (b) RFS, RBS and PF growth rates, relative to BBS growth rate, versus plasma density; the growth rates are calculated using a pump amplitude $a_{0}=2 a_{0-s c}$, for operation at lowest possible intensities while remaining in the strong-coupling regime. The plasma temperature is assumed to be $T_{e}=500 \mathrm{eV}$.

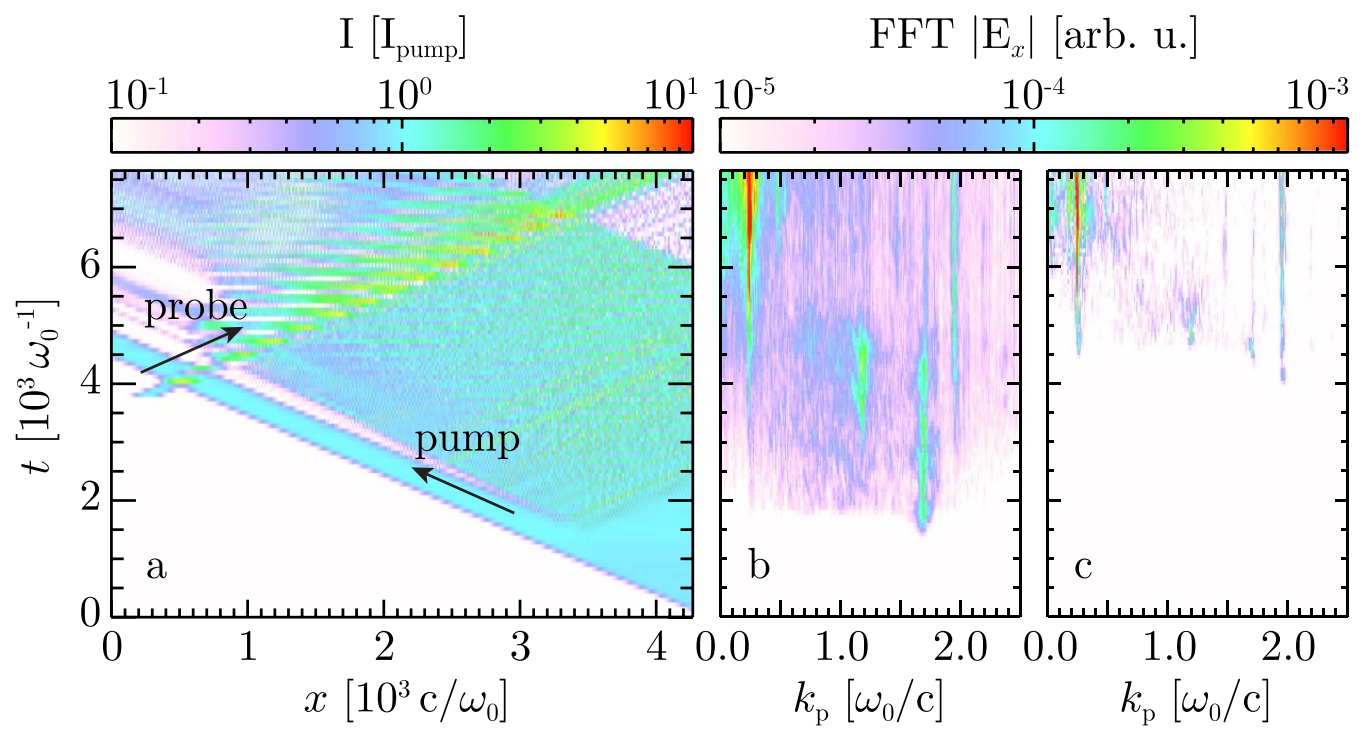

Figure 2. Example of pump-induced RBS/RFS and probe-induced RFS for $n_{0} / n_{c r}=0.05$ and pulse intensities of $10^{16} \mathrm{~W} \mathrm{~cm}{ }^{-2}$.

(a) Spatiotemporal evolution of laser intensity in the simulation domain (pump and probe pulses present). (b) Temporal evolution of Fourier components of electrostatic fluctuations for the simulation with pump and probe present. An additional simulation was performed without the probe pulse to capture the thermal scatter of the pump. This simulation was used to subtract the pump-induced electrostatic fluctuations from the simulation with both pump and probe, giving some insight into the isolated effects of the probe (c).

The numerical parameters for our simulations are as follows. In all simulations, we used 1000 particles per cell per species $\left(\sim 10^{9}\right.$ particles in total per simulation). The particles were shaped using a quadratic shape function. The cell size is given by $d x=\lambda_{0} / 95=2 \lambda_{D}$. The size of the simulation domain varied for different simulations. In general we chose the plasma column length to be several multiples of the scBrillouin growth length; the numerical experiments presented in the paper use plasma column lengths that range from 15 to $30 \mathrm{sc}$-Brillouin growth lengths $\left(v_{g} / \gamma_{B B S-s c}\right)$. The simulation domain size was thus chosen to be large enough to fully encompass the plasma column, as well as vacuum regions before and after the plasma column that corresponded to the initial length of the seed pulse.
The spatiotemporal evolution of laser intensity in the simulation domain is shown figure 2(a). The pump propagates in the negative $x_{1}$ direction, and thermal Raman backscatter is shown to develop before the pump meets the probe. The Langmuir wave at $k=k_{0}+\left(\omega_{0} / c\right) \sqrt{1-2 \omega_{p e} / \omega_{0}} \simeq 1.7 \omega_{0} / c$ associated with the pump-driven Raman backscatter is also visible in figure 2(b), which shows the Fourier spectrum of electrostatic fluctuations in the simulation domain. This process not only depletes the pump, reducing the amount of energy that can be transferred to the probe, but also leads to a strong prepulse that degrades the contrast of the main amplified probe pulse.

The pump eventually meets the counter-propagating probe pulse at $\omega_{0} t \simeq 4 \times 10^{3}$. During the initial stage of interaction, 

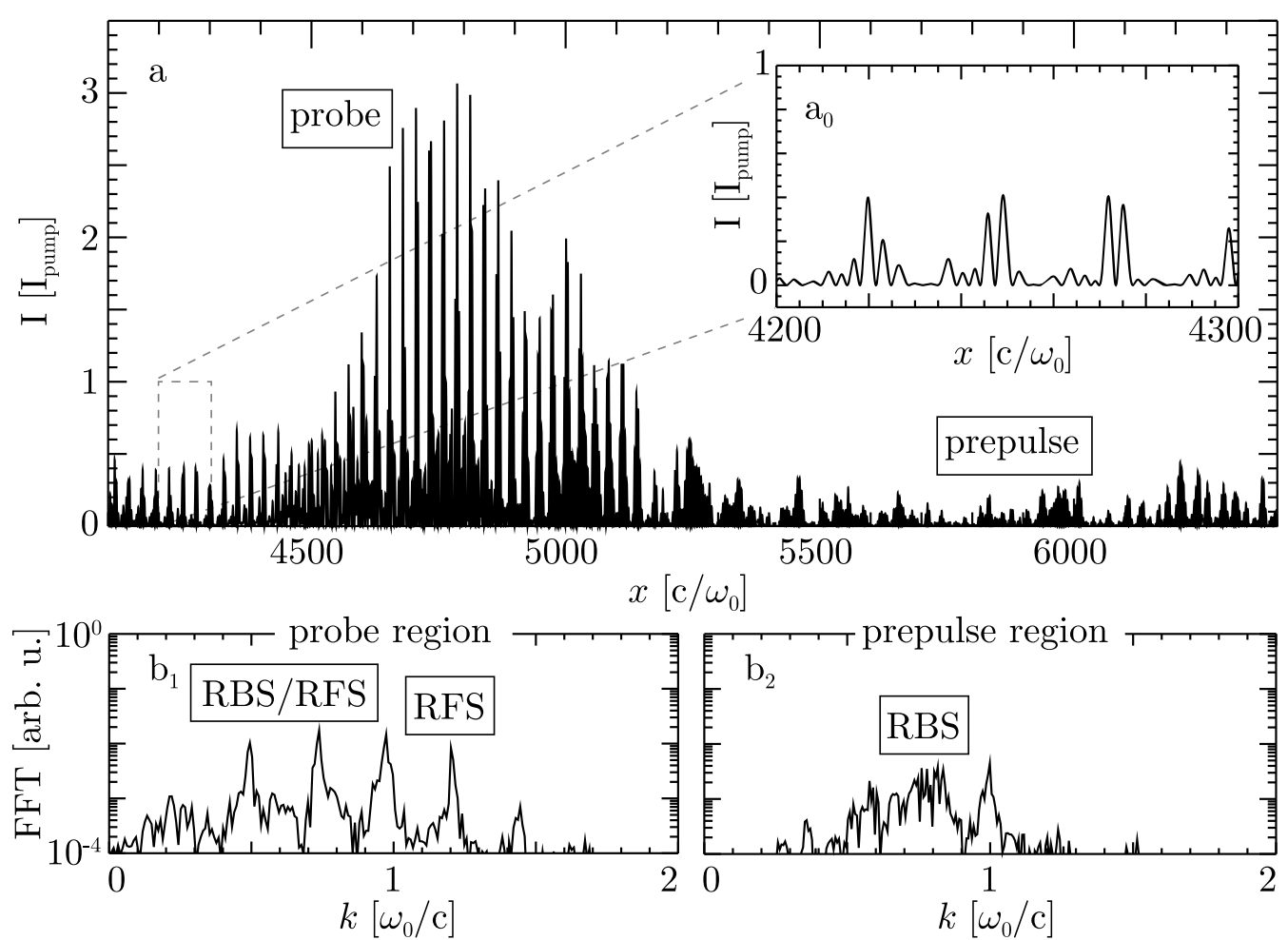

Figure 3. a) Longitudinal laser intensity profile at the exit of the plasma column, showing pump-induced RBS/RFS and probe-induced RFS for the same simulation as figure 2 . Inset $a_{0}$ reveals the development of incoherence at the probe tail, and insets $b_{1}$ and $b_{2}$ show the spectral signatures of the probe and prepulse regions, respectively.

around $\omega_{0} t \simeq 5 \times 10^{3}$, the probe pulse is able to efficiently absorb the energy of the pump, leaving almost no pump energy behind it. This time coincides with the emergence of the spectral signature of the ion plasma wave at $k \simeq 2 \omega_{0} / c$ in figure 2(b), indicating good Brillouin coupling. However, strong RFS is also triggered shortly after the probe enters the plasma, as seen in figure 2(b) at $k \sim \omega_{p e} / c \simeq 0.22 \omega_{0} / c$. In order to more clearly quantify the effect of the probe pulse on the excited plasma waves from the purely pump-induced thermal scattering, we have repeated the simulation without the probe pulse present, and subtracted the electrostatic fluctuations of the "pump-only" simulation from the "pump+probe" simulation, leading to the spectrum of electrostatic fluctuations presented in figure 2(c). Here it is clear that the spectral signatures at $k \simeq 1.7 \omega_{0} / c$ and $k \simeq 1.2 \omega_{0} / c$ are purely driven by the pump, and that the RFS and BBS modes are mainly determined by the presence of the probe, since the amplitude of their spectral signatures are very similar between figures 2(b) and (c). The RFS mode is observed to grow more than the BBS mode throughout the interaction, revealing a much stronger signature in the spectra of figures 2(b) and (c). This is correlated with the reduction in the Brillouin coupling between the pump and the probe, as most of the pump energy begins is transmitted behind the probe and not back-scattered, as shown in figure 2(a).

The intensity envelope of the amplified probe at the end of the interaction is shown in figure 3(a). A strong prepulse associated with thermal RBS by the pump is observed ahead of the amplified probe. In addition, the probe pulse envelope is found to be strongly modulated due to RFS, making RFS about as dangerous as filamentation at over-quarter-critical densities. A Fourier analysis of the $k$-spectrum of the pulses, shown in figures 3(b1) and (b2), confirms that the pump pulse mostly suffers from RBS, while RFS is dominant in the probe pulse. A close inspection of all Raman scattering occurring during Brillouin amplification reveals that the growth of the probe pulse saturates due to high levels of RFS, rather than Raman backscattering. If the level of RFS in the probe pulse becomes non-linear, the coherence of the probe pulse's carrier wave, and thus the coupling between pump and probe, is lost, and probe amplification stops. The probe decoherence due to RFS can be seen in figure 3(a0), and explains the decoupling between the pump and probe observed for $\omega_{0} t \gtrsim 5.5 \times 10^{3}$, as most of the pump energy is leaked behind the probe in figure 3(a).

Since the growth of RFS is reduced for decreasing plasma density, as shown in figure 1(b), it follows that the saturation of the probe pulse should be strongly affected by the plasma density, and that lowering this density even further is expected to improve the pump-to-probe amplification ratio and energy transfer. We have therefore simulated Brillouin amplification at a lower plasma density of $n_{0} / n_{c r}=0.01$, and also using lower pulse intensities of $10^{15} \mathrm{~W} / \mathrm{cm}^{2}$, corresponding to $a_{0}=0.027 \simeq 1.6 a_{0-s c}$. The plasma column length was adjusted such that we have maintain 15 strong-coupling Brillouin growth lengths $\left(v_{g} / \gamma_{B B S-s c}\right)$, as was considered in the previous case, corresponding to $L_{p}=10900 \mathrm{c} / \omega_{0} \simeq 1.7 \mathrm{~mm}$. The initial probe pulse duration was set to $\tau_{o p t}$, according to equation. 2 , 


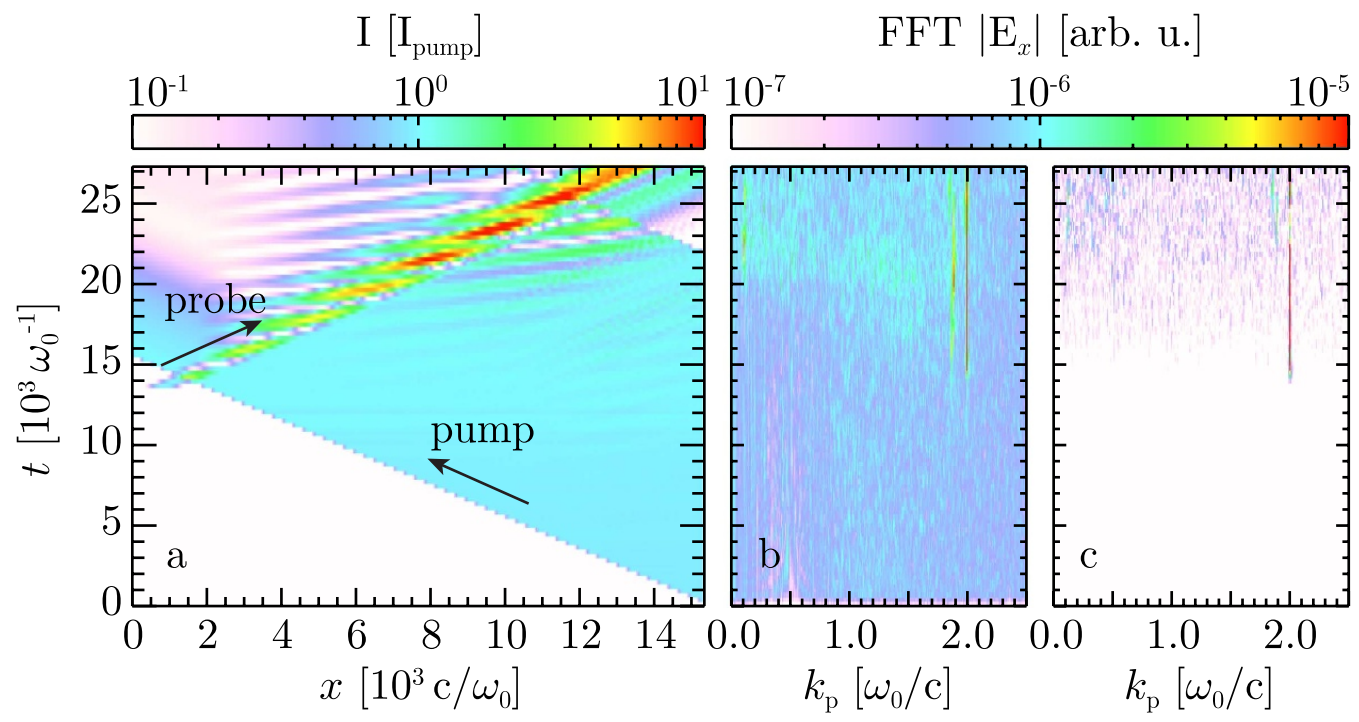

Figure 4. Example of successfully amplified probe for $n_{0} / n_{c r}=0.01$ and pulse intensities of $10^{15} \mathrm{~W} \mathrm{~cm}^{-2}$. (a) Spatiotemporal evolution of laser intensity in the simulation domain (pump and probe pulses present). (b) Temporal evolution of Fourier components of electrostatic fluctuations for the simulation with pump and probe present. An additional simulation was performed without the probe pulse to capture the thermal scatter of the pump. This simulation was used to subtract the pump-induced electrostatic fluctuations from the simulation with both pump and probe, giving some insight into the isolated effects of the probe (c).

and the pump duration was set to $\tau_{\text {pump }}=2 L_{p} / v_{g} \simeq 11.6 \mathrm{ps}$. The results are displayed in figures 4 and 5.

Figure 4(a) shows that thermal RBS by the pump is greatly reduced, as expected by the lower plasma density and pulse intensities, registering no thermal emission before meeting the probe. The probe enters the plasma at $\omega_{0} t \gtrsim 15 \times 10^{3}$ and, after a very short transient time, and couples to the pump wave through BBS, efficiently depleting the pump energy behind it. The spectral signature associated with the ion plasma wave at $k \simeq 2 \omega_{0} / c$ is clearly visible in both figures $4(\mathrm{~b})$ and (c), which again correspond to the spectra of electrostatic fluctuations of the "pump+probe" simulation and the subtraction between the "pump+probe" and "pump only" simulations, respectively. During the amplification process, however, some thermal RBS emission due to the pump is registered in figure 4(a) ahead of the amplifying probe, still compromising the contrast of the output pulse. The emergence of the RBS Langmuir wave is also visible in figure $4(\mathrm{~b})$ at $k=1.89 \omega_{0} / c$. Although this Langmuir wave seems to appear after the probe has entered the plasma, it is clear in the spectrum of figure 4(c) (which isolates the effects of the probe) that presence of the probe pulse is not responsible for the growth of the Langmuir wave. Nevertheless, good Brillouin amplification is observed, owing mainly to the strong reduction in RFS, which shows only a very weak spectral signature towards the end of the interaction at $k \simeq \omega_{p e} / c=0.1 \omega_{0} / c$ in figures $4(\mathrm{~b})$ and (c).

The intensity envelope of the amplified probe pulse is presented in figure 5(a), showing a much weaker prepulse, due to the reduced thermal RBS by the pump, and a much smoother longitudinal envelope due to the reduced RFS. The spectral content of the probe and prepulse are respectively shown in figures 5(b1) and (b2), verifying the above interpretation.

The improvement in the Brillouin amplification performance between the simulations at $n_{0} / n_{c r}=0.05$ and $n_{0} / n_{c r}=$
0.01 is mainly due to the reduction in RFS. It is therefore clear that probe decoherence due to RFS is more dangerous than pump depletion due to thermal RBS. Note, however, that there can be an additional interplay between pump RBS and probe RFS: thermal RBS driven by the pump can seed and stimulate the growth of RFS in the probe. When the probe pulse $\left(\omega_{0}, k_{0}\right)$ overlaps with the thermal RBS emission from the pump $\left(\omega_{0}-\omega_{p e}, k_{0}-\omega_{p e} / c\right)$, the resulting beat wave excites a Langmuir wave with $\left(\omega_{p e}, \omega_{p e} / c\right)$, which follows the probe pulse. In turn, the beating between this Langmuir wave and the probe pulse can trigger the emission of light at $\left(\omega_{0}+\omega_{p e}, k_{0}+\omega_{p e} / c\right)$, i.e. RFS, whose nonlinear development will ultimately destroy the coherence of the probe, and shut down the Brillouin amplification process. This is a consequence of both pump and probe pulses having very close frequencies $\left(\omega_{0}\right)$, when attempting Brillouin amplification at subquarter-critical densities, and can become more important with increasing interaction length.

In order to further investigate the density range over which Brillouin amplification performs best, we have carried out additional 1D PIC simulations for plasma densities of $n_{0} / n_{c r}=0.001,0.005,0.01,0.05$ and 0.1 , and using pulse intensities corresponding to $2 a_{0-s c}$, just above the strongcoupling threshold. The length of the plasma column in each case was adjusted such that $\gamma_{B B S-s c} L_{p} / v_{g}=30$; the results are summarized in table 1 .

Despite using the lowest possible pulse intensities, while still satisfying the strong-coupling Brillouin threshold, the simulations for the highest plasma densities of $n_{0} / n_{c r}=0.05$ and 0.1 were still plagued by probe RFS and pump RBS, compromising the quality of the probe pulse. Good amplification results were obtained for the lower-density simulations, owing to the reduction in parasitic Raman scattering. RFS and RBS levels are further reduced at lower plasma densities, but so 

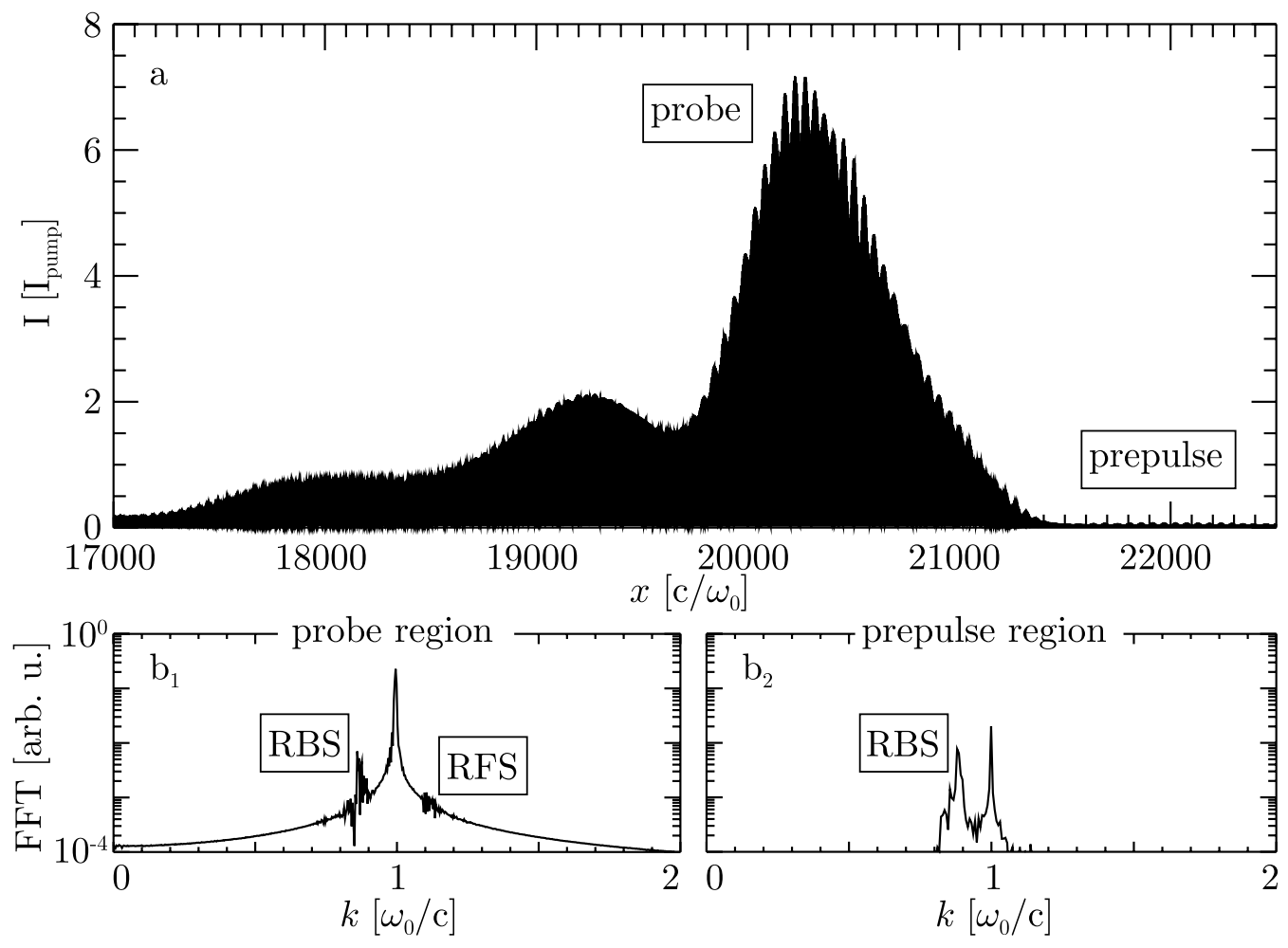

Figure 5. (a) Longitudinal laser intensity profile at the exit of the plasma column for the same simulation as figure 4. For this case, the pulse envelope is significantly smoother and the prepulse intensity much lower than the case shown in figure 4(a), even though the interaction time is five times longer. Insets $b_{1}$ and $b_{2}$ show the spectral signatures of the probe and prepulse regions, respectively.

Table 1. Brillouin amplification results for different (sub-quarter-critical) densities at pump/probe pulse intensities just above the strong-coupling threshold, $a_{0}=2 a_{0-s c}$. Displayed are the results for the amplification ratio $R_{A} \equiv I_{f} / I_{0}$, where $I_{f}$ is the intensity of the amplfied probe pulse, compression ratio $R_{C} \equiv \tau_{f} / \tau_{\text {pump }}$, and energy transfer efficiency $\eta$. Results for $n_{0} / n_{c r}=0.05$ and 0.1 are not presented because the interaction was dominated by the severe development of RBS and RFS instabilities, having destroyed the temporal profile of the amplified probe pulse.

\begin{tabular}{llllll}
\hline & \multicolumn{5}{c}{$n_{0} / n_{c r}$} \\
\cline { 2 - 6 } & 0.001 & 0.005 & 0.01 & 0.05 & 0.1 \\
\hline$R_{A}$ & 1.4 & 3.6 & 7.5 & RFS/RBS & RFS/RBS \\
$R_{C}$ & 33 & 9 & 14 & RFS/RBS & RFS/RBS \\
$\eta$ & $<1 \%$ & $35 \%$ & $48 \%$ & RFS/RBS & RFS/RBS \\
\hline
\end{tabular}

is the coupling between the probe and pump pulses through the plasma, reducing the energy transfer efficiency. The best amplification performance was obtained for $n_{0} / n_{c r}=0.01$, having reached amplification and compression ratios of 7.5 and 14 , respectively, and absorbing $48 \%$ of the energy of the pump (in the leading peak of the probe). At $n_{0} / n_{c r}=0.005$, good energy transfer efficiency was also observed, reaching $\eta=35 \%$ and amplification and compression ratios of 3.6 and 9 , respectively. At $n_{0} / n_{c r}=0.001$, the plasma density was too low for any significant coupling between the pump and probe pulses $(\eta<1 \%)$, so no significant probe amplification could be observed.

RFS of the probe pulse is not just enhanced by higher plasma densities, but also by higher probe intensities. It may therefore frustrate our aim to amplify the probe pulse to the highest possible intensities. Probe RFS puts an upper limit on the compression and amplification ratios that can be reached for sub-quarter-critical densities. For example, it was shown that a probe pulse could be amplified from $10^{16} \mathrm{~W} \mathrm{~cm}^{-2}$ to $10^{17} \mathrm{~W} \mathrm{~cm}^{-2}$ and from $10^{17} \mathrm{~W} \mathrm{~cm}^{-2}$ to $5 \times 10^{17} \mathrm{~W} \mathrm{~cm}^{-2}$ in two separate simulations at $n_{0} / n_{c r}=0.05$ [27, 28]. However, if one wishes to combine these two stages to obtain amplification from $10^{16} \mathrm{~W} \mathrm{~cm}^{-2}$ to $5 \times 10^{17} \mathrm{~W} \mathrm{~cm}^{-2}$ in a two stage experiment, then RFS growth in the first stage needs to be tightly controlled. Otherwise the probe RFS generated during the first stage may well saturate the amplification during the second stage before an intensity of $5 \times 10^{17} \mathrm{~W} \mathrm{~cm}^{-2}$ is reached.

As shown above, lowering the plasma density will immediately improve the pump-to-probe amplification ratio and energy transfer, but it may also reduce the Brillouin backscattering growth rate, especially at the beginning of the interaction when the probe intensity is still low. Using a plasma density profiles with a 'ramp' rather than a 'plateau', with the 

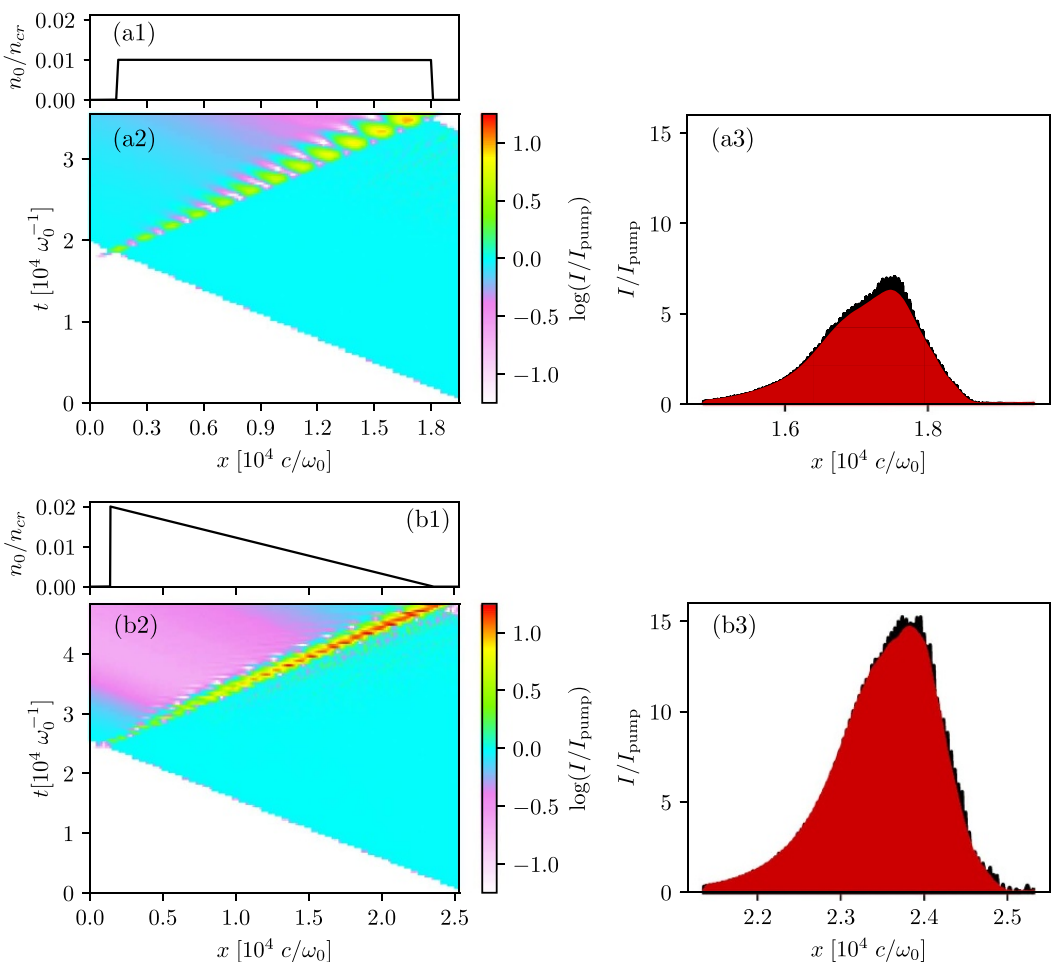
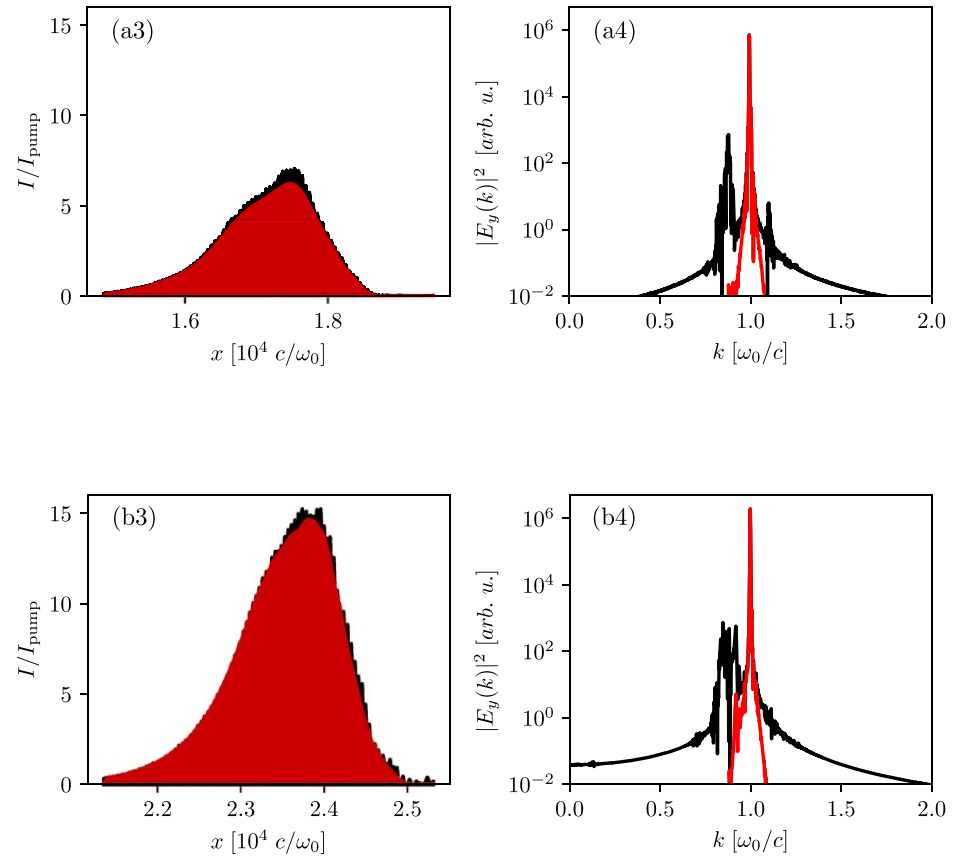

Figure 6. Comparison of Brillouin amplification using a 'plateau' density profile (a1-a4) and a 'ramp' density profile (b1-b4). Shown are the initial plasma density profiles (a1, b1), the intensity amplification factor as a function of $x$ and $t(\mathrm{a} 2, \mathrm{~b} 2)$, the final raw (black) and filtered (red) intensity envelopes of the probe pulses $(\mathrm{a} 3, \mathrm{~b} 3)$ and the Fourier power spectra of the raw (black) and filtered (red) probe pulses. The bandpass filter on the amplified probe pulse captures frequencies close to $\omega_{0}$, which is the Brillouin-amplified frequency, and cleans up the contributions from parasitic Raman. We find that the 'ramp' profile leads to a higher amplification factor and a smoother pulse envelope than the 'plateau' profile, which is likely caused by the lower levels of stimulated Raman scattering seen in the power spectrum of the 'ramp' case.

highest plasma density facing the probe pulse, as proposed by $[27,28]$, could be a good compromise in this case. To investigate this, we compared two simulations, one with a rectangular 'plateau' density profile and one with a continuous downward density ramp. The density profiles were chosen such that the average density for both cases is the same, to make the comparison fairer. In both simulations, the average density is $n_{0} / n_{\mathrm{cr}}=$ 0.01 . In both cases, the density profile extends for 30 Brillouin growth lengths; this causes the 'ramp' density profile to have a slightly longer extent than the 'plateau' profile. The amplitudes of both pump and probe pulse are given by $a_{0}=a_{1}=$ $1.5 a_{0-s c}$, where $a_{0-s c}$ is calculated for a plasma temperature of $250 \mathrm{eV}$ and an average density of $n_{0}=0.01 n_{c r}$. The results of the comparison are displayed in figure 6. For the 'plateau' case, we find that the pump-to-probe amplification factor is 7 , while the compression factor is 22 , leading to an efficiency of 0.31 . For the 'ramp' case, we find an amplification factor of 15 and a compression factor of 37 , leading to an efficiency of 0.41. From figure 6, frames (a1), (b1), (a3) and (b3), we find that this likely follows from the fact that the 'ramp' profile has a higher density on the left, i.e. at the beginning of the interaction between pump and seed pulses, which kick-starts the amplification process. From frames (a4) and (b4), we find that the 'ramp' case also shows a lower level of thermal Raman scattering (both backward and forward) compared to the "plateau' case, although this appears to have only a minor influence on the end result. In order to minimize SRS from thermal noise in the plasma, one could also aim to reduce the plasma electron temperature during the interaction, i.e. reduce SRS seeding.

In figure 6, frames (a3) and (b3), we show the final raw (black) and filtered (red) intensity envelopes of the probe pulses, where the filter removes contributions of parasitic Raman scattering from the amplified probe pulse. The two envelopes are very close to each other, which proves that the vast majority (about 99\%) of the energy contained in the probe pulse is due to Brillouin amplification, and that the Raman contribution to the seed energy content is negligible. As demonstrated by Jia et al [29], it is important to distinguish between contributions from Raman and Brillouin scattering when studying Brillouin amplification at plasma densities below $n_{c r} / 4$, where both processes occur simultaneously and are often competing.

It should be noted that frequency matching between pump, probe and ion-acoustic frequencies is not really an issue in these simulations, even for position-dependent density profiles such as the 'plateau' or the 'ramp', since the ion-acoustic frequency is so small that the frequency difference between the pump and probe pulses is always fully covered by the bandwidth of the probe pulse. Conversely, the non-constant plasma density and electron plasma frequency may strongly impact 
the growth of all forms of Raman scattering, which is rather beneficial in this case.

Finally, we comment on those transverse effects that will occur in multi-dimensional settings, but which are not covered in our one-dimensional simulations. The two most important transverse effects in 2D/3D scenarios are: (1) the bow-shaped transverse structure of the amplified seed [9, 26, 39] and (2) filamentation/self-focusing of the seed [17, 19, 36-38]. Of these effects, the transverse bowing will always be there, as it is an inherent feature of the Brillouin amplification process [26], while self-focusing and filamentation are separate effects, which can be mitigated or suppressed for the right combination of laser and plasma parameters. We note that filamentation is not the most important concern at the plasma densities we use in this paper $\left(0.05 n_{c r}\right)$. Nevertheless, other works which have focused on Brillouin amplification at plasma densities larger than $0.25 n_{c r}$ where filamentation is strong, e.g. Zhang et al [39] or Alves et al [9], have shown that it can be suppressed or at least controlled via tuning of the laser and plasma parameters. Self-focusing in the context of Raman amplification has been shown to mostly affect tightly focused seed pulses after long interaction lengths [19]. For Brillouin amplification of wide pulses to moderate peak intensity, the typical distance for self-focusing can be made larger than the interaction distance, which mostly eliminates this process. Fluid/envelope models can qualitatively capture all these transverse effects, as has been demonstrated in the past (see e.g. the discussion on bow-shaped seed pulses in Trines et al [26], or the work by Li et al for filamentation [40]). However, such models may fail in the nonlinear regime of the amplification, where plasma waves can reach highamplitudes and develop sharp gradients. In the presence of sharp gradients, the fluid/envelope equations can break down, requiring a fully kinetic treatment via multi-dimensional PIC simulations. This will be the subject of future studies in this field.

\section{Conclusions}

We have studied Brillouin amplification of short laser pulses in plasma at electron densities $n_{0} / n_{c r}<0.25$. At such densities, filamentation of the growing probe laser pulse is reduced compared to e.g. $n_{0} / n_{c r}=0.3$, but stimulated Raman scattering, which is inhibited for $n_{0} / n_{c r}>0.25$, suddenly becomes possible and introduces extra complications. Raman backscattering of the pump pulse adds a large pre-pulse to the amplified probe, while RFS of the probe itself causes strong envelope modulations and a reduction of pulse quality. Even worse, non-linear RFS destroys the coherence of the probe pulse's carrier wave, inhibiting further Brillouin amplification. Therefore, parasitic Raman scattering needs to be reduced at all cost in order to boost Brillouin amplification at sub-quarter-critical plasma densities. Recent works have explored SRS mitigation strategies through manipulation of the pump/probe frequency chirp [23, 25] and the addition of external magnetic fields $[30,31]$. Here we have shown how SRS can be minimized during Brillouin amplification by appropriately designing the density profile of the plasma column.

Fortunately, the RFS growth rate scales much faster with the plasma density than the BBS growth rate $\left(n_{0}\right.$ versus $\left.n_{0}^{1 / 3}\right)$, so reducing the plasma density will immediately reduce RFS levels without significantly compromising the Brillouin amplification process. We have performed a range of 1-D PIC simulations where we varied the pump laser intensity, the plasma density and the interaction length. The simulation results showed that lowering either the plasma density or the pump intensity led to a significant improvement in the amplification and compression ratios, as well as the quality of the amplified pulse. The best result obtained was for $n_{0} / n_{c r}=0.01$ and a pump intensity of $10^{15} \mathrm{~W} \mathrm{~cm}^{-2}$, although there are strong indications that even better results can be obtained by increasing the interaction length for the simulations at $10^{14} \mathrm{~W} \mathrm{~cm}^{-2}$ pump intensity and $n_{0} / n_{c r}=0.01$. In particular, we conclude that Brillouin amplification should be conducted at densities for which RFS is either impossible $\left(n_{0} / n_{c r}>0.25\right)$ or unimportant $\left(n_{0} / n_{c r} \leq 0.01\right)$. For $0.01<$ $n_{0} / n_{c r}<0.25$, the disadvantage of increased pump RBS and probe RFS is more serious than the advantage of reduced probe filamentation.

As a compromise between using a higher density to improve Brillouin scattering and a lower density to reduce Raman scattering, one can use a plasma density profile with a 'ramp' rather than a 'plateau', with the highest plasma density facing the probe pulse. This will stimulate Brillouin scattering during the early stages of the interaction, when the probe intensity is still low, while reducing RFS later on, when the probe intensity is much higher. Initial simulations of this scenario showed a reduction in RFS accompanied by an improvement in probe amplification and quality, so the use of tailored plasma density profiles deserves further investigation. In particular, one could aim to combine the strengths of the 'plateau' and 'ramp' profiles by using a 'trapezoid' profile.

\section{Data availability statement}

The data that support the findings of this study are available upon reasonable request from the authors.

\section{Acknowledgments}

This work was supported by the STFC Central Laser Facility, the STFC Centre for Fundamental physics and by EPSRC through Grant EP/G04239X/1. We would like to thank R Kirkwood for stimulating discussions and the OSIRIS consortium for the use of OSIRIS. We acknowledge PRACE for providing access to the resource SuperMUC based in Germany at the Leibniz Research Center. LOS acknowledges the support of the European Research Council (ERC-2015-AdG Grant No. 695088). Simulations were performed on the ScarfLexicon Cluster (STFC RAL), the IST Cluster (IST Lisbon) and SuperMUC (Leibniz Supercomputing Centre, Garching, Germany). 


\section{ORCID iDs}

R M G M Trines (D) https://orcid.org/0000-0003-2553-0289

E P Alves (D) https://orcid.org/0000-0002-4588-1003

R Bingham (D) https://orcid.org/0000-0002-9843-7635

R A Cairns (D) https://orcid.org/0000-0002-7562-2053

F Fiúza (D) https://orcid.org/0000-0002-8502-5535

R A Fonseca (D) https://orcid.org/0000-0001-6342-6226

L O Silva (D) https://orcid.org/0000-0003-2906-924X

\section{References}

[1] Maier M, Kaiser W and Giordmaine J A 1966 Phys. Rev. Lett. 171275

[2] Milroy R D, Capjack C E and James C R 1977 Plasma Phys. 19989

[3] Milroy R D, Capjack C E and James C R 1979 Phys. Fluids 221922

[4] Capjack C E, James C R and McMullin J N 1982 J. Appl. Phys. 534046

[5] Andreev A A and Sutyagin A N 1989 Sov. J. Quantum Electron. 191579

[6] Malkin V M, Shvets G and Fisch N J 1999 Phys. Rev. Lett. 824448

[7] Andreev A A et al 2006 Phys. Plasmas 13053110

[8] Forslund D W, Kindel J M and Lindman E L 1975 Phys. Fluids 18 1002-16

[9] Alves E P et al 2021 Plasma Phys. Control. Fusion 63114004

[10] Kirkwood R et al 1999 Phys. Rev. Lett. 832965

[11] Ping Y et al 2004 Phys. Rev. Lett. 92175007

[12] Ren J et al 2007 Nat. Phys. 3 732-6

[13] Ping Y et al 2009 Phys. Plasmas 16123113

[14] Lancia L et al 2010 Phys. Rev. Lett. 104025001

[15] Lancia L et al 2016 Phys. Rev. Lett. 116075001
[16] Marquès J-R et al 2019 Phys. Rev. X 9021008

[17] Trines R M G M et al 2011 Nat. Phys. 7 87-92

[18] Kirkwood R K et al 2011 Phys. Plasmas 18056311

[19] Trines R M G M et al 2011 Phys. Rev. Lett. 107105002

[20] Toroker Z, Malkin V M and Fisch N J 2012 Phys. Rev. Lett. 109085003

[21] Lehmann G and Spatschek K H 2013 Phys. Rev. E 87063107

[22] Lehmann G and Spatschek K H 2013 Phys. Plasmas 20073112

[23] Lehmann G and Spatschek K H 2015 Phys. Plasmas 22043105

[24] Chiaramello M et al 2016 Phys. Plasmas 23072103

[25] Chiaramello M, Amiranoff F, Riconda C and Weber S 2016 Phys. Rev. Lett. 117235003

[26] Trines R M G M et al 2020 Sci. Rep. 1019875

[27] Weber S et al 2013 Phys. Rev. Lett. 111055004

[28] Riconda C et al 2013 Phys. Plasmas 20083115

[29] Jia Q, Ido B, Edwards M R, Mikhailova J M and Fisch N J 2016 Phys. Plasmas 23053118

[30] Edwards M R, Yuan S, Mikhailova J M and Fisch N J 2019 Phys. Rev. Lett. 123025001

[31] Zhaoli Li, Zhaohui W, Zuo Y, Zeng X, Wang X, Wang X, Jie M, Bilong $\mathrm{H}$ and Jingqin S 2021 Phys. Plasmas 28013107

[32] Kim J, Lee H J, Suk H and Ko I S 2003 Phys. Lett. A 314464

[33] Fonseca R A et al 2002 Lect. Notes Comput. Sci. 2331 342-51

[34] Fonseca R A et al 2003 Phys. Plasmas 101979

[35] Fonseca R A et al 2008 Plasma Phys. Control. Fusion 50124034

[36] Kaw P, Schmidt G and Wilcox T 1973 Phys. Fluids 161522

[37] Bingham R and Lashmore-Davies C N 1976 Nucl. Fusion 1667

[38] Max C E, Arons J and Langdon A B 1974 Phys. Rev. Lett. 33209

[39] Zhang Z M, Zhang B, Hong W, Deng Z G, Teng J, He S K, Zhou W M and Gu Y Q 2017 Phys. Plasmas 24113104

[40] Li Z et al 2019 Phys. Plasmas 26093102 